\title{
Effect of Benzoic Acid on the Metabolic Conjugations of p-Aminobenzoic Acid in the Rat
}

\author{
MARTTI KOIVUSALO, TAPAN I LUUKKAINEN, \\ TATU MIETTINEN and JAAKKO PISPA \\ Department of Medical Chemistry, University of Helsinki, Finland
}

\begin{abstract}
The excretion of $p$-aminobenzoic acid and its conjugates is determined by the counter current distribution technique in rat urine after the administration of $p$-aminobenzoic acid alone and with the addition of benzoate.

It has been found that $p$-aminobenzoic acid is excreted also in rat urine as glucuronide in addition to acetyl and glycine conjugates.

After administration of benzoate with $p$-aminobenzoic acid the amounts of glycine and glucuronide conjugates decreased whereas the amount of acetyl-p-aminobenzoic acid increased.

Only a small fraction of the administered $p$-aminobenzoic acid was excreted as such and this was unaffected by benzoate.
\end{abstract}

M ost of the administered $p$-aminobenzoic acid is rapidly excreted by the mammal in the urine as different conjugates and only a small part is excreted as free $p$-aminobenzoic acid ${ }^{1-3}$. In man most of the administered $p$-aminobenzoic acid is conjugated with glycine to $p$-aminohippurate, but also appreciable amounts of acetyl-p-aminobenzoate and of the glucuronides of $p$-aminobenzoic acid and acetyl- $p$-aminobenzoic acid are excreted with small amounts of free $p$-aminobenzoic acid and acetyl- $p$-aminohippurate ${ }^{3,4}$. Similar results have been obtained in rabbits ${ }^{5-7}$. In rat urine Riggs and Christensen ${ }^{8}$ found $p$-aminohippurate, $p$-aminobenzoate and their acetyl derivatives after administration of $p$-aminobenzoic acid. Simultaneous administration of benzoic acid increased the amount of acetyl- $p$-aminobenzoate and decreased the amount of glycine conjugates excreted ${ }^{8}$.

In the present paper results are reported from studies by the counter current distribution technique on the excretion pattern of $p$-aminobenzoic acid and its metabolites in rat urine. It was found that also in rats there was an appreciable excretion of glucuronide conjugates, as had been found earlier in man and in rabbits. Simultaneous administration of benzoic acid abolished almost totally the excretion of conjugates other than acetyl- $p$ aminobenzoate whose excretion increased correspondingly. The excretion of unconjugated $p$-aminobenzoate was unaffected by the administration of benzoate.

Acta Chem. Scand. 12 (1958) No. 10 


\section{EXPERIMENTAL}

The experimental animals were 40 white rats of Wistar strain weighing $180-220 \mathrm{~g}$. The diet during the experiments was the usual stock diet of this Department.

The dosage of $p$-aminobenzoic acid was $15 \mu$ moles per rat and it was given in $5 \mathrm{ml}$ of water by stomach tube. Urine was collected without preservative during a period of $4 \mathrm{~h}$ after the administration of $p$-aminobenzoic acid and the urine samples of all rats were pooled. After five days the same rats were given $300 \mu$ moles of sodium benzoate and $15 \mu$ moles of $p$-aminobenzoic acid in $5 \mathrm{ml}$ of water and the urines collected as above.

The counter current distribution technique of Craig ${ }^{9}$ was used. The solvent system was amyl alcohol and $0.1 \mathrm{M}$ acetate buffer $\mathrm{pH} 3.4,24$ transfers were made in an all-glass apparatus.

The glucuronides were determined in the aqueous phase by the modified naphthoresorcinol method of Miettinen et al. ${ }^{10}$ Before analysis the samples were extracted three times with ether to remove the traces of amyl alcohol present.

The diazotisable amines were determined by the method of Bratton and Marshall 11 before and after acid hydrolysis. The determinations were made from both phases. $p$-Aminobenzoic acid and its derivatives were extracted from the organic phase with $0.1 \mathrm{~N} \mathrm{NaOH}$

The distribution coefficients of authentic samples of $p$-aminobenzoic acid, $p$-aminohippuric acid and acetyl-p-aminobenzoic acid were determined in the solvent system used, and compared with those obtained from the peaks of the experimental distribution curves uring the formula presented by Rauen and Stamm ${ }^{12}$. These values are presented in Table 1 and the identification of the peaks in the distribution curves is based on them.

Table 1. Distribution coefficients of $p$-aminobenzoic acid and its conjugates in amyl alcohol/acetate buffer.

$\begin{array}{lcc} & \text { Determined } & \begin{array}{c}\text { Calculated from } \\ \text { the curves }\end{array} \\ p \text {-Aminobenzoic acid } & 10.80 & 11.00 \\ \text { Acetyl-p-aminobenzoic acid } & 14.00 & 15.67 \\ p \text {-Aminohippuric acid } & 0.36 & 0.35 \\ \text { Acetyl-p-aminohippuric acid } & - & 1.00\end{array}$

\section{RESULTS AND DISCUSSION}

The results are presented in Fig. 1 and 2 . It is seen from Fig. 1 that in the normal rat most of the $p$-aminobenzoic acid excreted in the urine is conjugated with glycine to form $p$-aminohippurate and acetyl- $p$-aminohippurate. An appreciable amount of acetyl- $p$-aminobenzoate is also excreted but the amount of free $p$-aminobenzoate is rather small. The $p$-aminobenzoic acid glucuronides excreted are mostly unconjugated as regards the amino group, only negligible amounts of acetyl- $p$-aminobenzoic acid glucuronide were found. The glucuronide peak apparently contains also other glucuronides besides those of $p$-aminobenzoic acid and its derivatives, as is seen when the total amount of amines in the first plates is compared with the amount of total glucuronide in the same plates.

Conversely, when benzoate was also administered, the excretion of the glycine conjugates of $p$-aminobenzoic acid fell to very low values, but was not totally abolished. No acetyl- $p$-aminobenzoic acid glucuronide was found and the amount of $p$-aminobenzoic acid glucuronide was also much diminished. The 

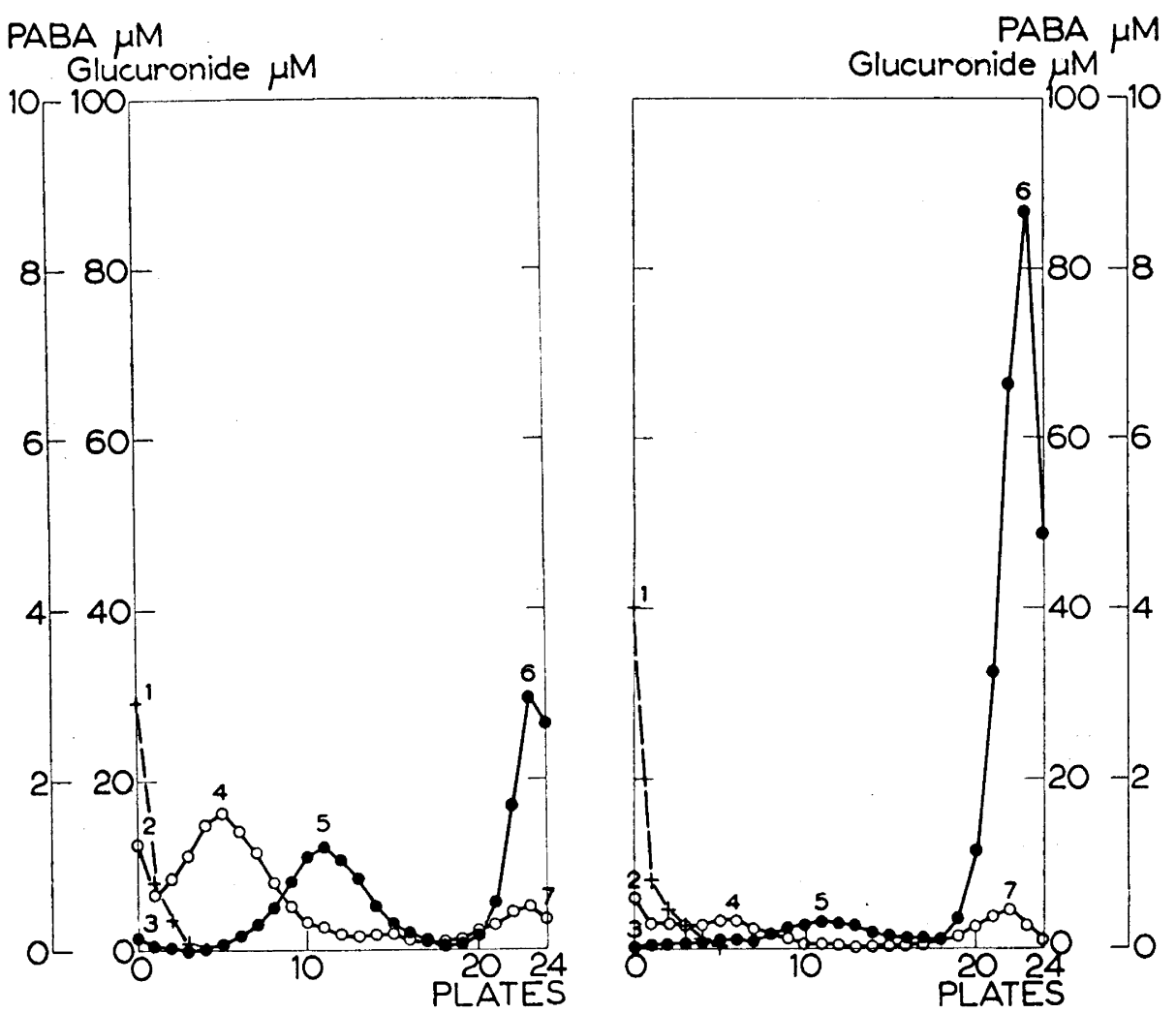

Fig. 1. Excretion pattern of $p$-aminobenzoic acid and its conjugates in rat urine.

+-+ Glucuronide $\mathrm{O}-\mathrm{O}$ Diazotisable amines

- Undiazotisable amines acid glucuronide. 4. $p$-Aminohippurate. 5. Acetyl-p-aminohippurate. 6. Acetyl-paminobenzoate. 7. $p$-Aminobenzoate.

Fig. 2. Excretion pattern of $p$-aminobenzoic acid and its conjugates after simultaneous administration of benzoate.

The curves and peak numbers as in Fig. 1.

total amount of glucuronides was increased probably due to the excretion of benzoic acid glucuronide. The excretion of acetyl- $p$-aminobenzoic acid was correspondingly increased, it was in fact the only metabolite of $p$-aminobenzoic acid excreted in the urine in noteworthy amounts after administration of benzoate. The low excretion of unconjugated $p$-aminobenzoic acid was not affected by benzoate.

The appreciable amounts of $p$-aminobenzoic acid glucuronides excreted by the rat makes it necessary to determine also these conjugates in addition to

Acta Chem. Scand. 12 (1958) No. 10 
glycine and acetyl conjugates when the pattern of the $p$-aminobenzoic acid conjugates is determined in the urine. If this is not done, the amounts of free $p$-aminobenzoic acid and $p$-aminohippuric acid in particular will be estimated too high.

The relative amount of acetyl conjugates calculated as a percentage of the total $p$-aminobenzoic acid excretion increased after administration of benzoate from 53 to $85 \%$. This rise is not due to any activation of the acetylation processes but presumably to the inhibition of the glycine conjugation, which in turn leads to diminished excretion of total $p$-aminobenzoic acid as shown by the experiments on the acetylation of aromatic amines by Luukkainen ${ }^{13}$.

An interesting observation is that, although the excretion pattern of all the conjugates is changed after the administration of benzoate, the amount of unconjugated $p$-aminobenzoic acid excreted remained constant.

\section{REFERENCES}

1. Beyer, K. H., Tillson, E. K., Runo, H. F., Schuchardt, G. S. and Pitt, A. A. Federation Proc. 11 (1952) 13.

2. Lustig, B., Goldfarb, A. R. and Gerstl, G. Arch. Biochem. 5 (1944) 59.

3. Tabor, C. W., Freeman, M. F., Baily, J. and Smith, P. K. J.Pharmacol. Exptl. Therap. 102 (1951) 98.

4. Mandel, H. G., Yen, C. and Smith, P. K. Federation Proc. 11 (1952) 372.

5. Bray, H. G., Lake, H. J., Neale, F. C., Thorpe, W. V. and Wood, P. B. Biochem. J. 42 (1948) 434.

6. Umeda, Y. Fukuoka-Jgaku-Zasshi 48 (1957) 299; Chem. Abstracts 52 (1958) 2228.

7. Venkataraman, P. R., Venkataraman, A. and Lewis H. B. Arch. Biochem. Biophys. 26 (1950) 173.

8. Riggs, T. R. and Christensen, H. N. J.Biol.Chem. 193 (1951) 675.

9. Craig, L. C. J.Biol.Chem. 155 (1944) 519.

10. Miettinen, T., Ryhänen, V. and Salomaa, H. Ann. Med. exptl. et Biol. Fenniae, Helsinki 35 (1957) 173.

11. Bratton, A. C. and Marshall, E. K., Jr. J.Biol.Chem. 128 (1939) 537.

12. Rauen, H. M. and Stamm, W. Gegenstromverteilung, Springer-Verlag, Würzburg 1953 , p. 46.

13. Luukkainen, T. Acta Physiol. Scand. 45 (1956) Suppl. 154.

Received August 28, 1958. 